\title{
Erdheim-Chester Disease
}

\author{
${ }^{1}$ Mamatha Patil, ${ }^{2}$ Deepak Jaiswal
}

\begin{abstract}
Erdheim-Chester disease (ECD) is an extremely rare disorder that can affect many different organs of the body. This is an unusual form of non-Langerhans-cell histiocytosis. This is characterized by excessive production and accumulation of histiocytes. The normal function of the histiocytes is to fight infections. Histiocytes accumulate in the loose connective tissue of various organ systems of the body and cause thickening of tissues and may become dense and fibrotic. In the absence of successful treatment, the disease is debilitating and can result in multi-system organ failure. Erdheim-Chester disease is often described in the medical literature as an extremely rare ${ }^{1}$ entity. Erdheim-Chester disease usually presents in adults aged between 40 and 60 years. Here we present a case report of ECD in a 26 -year-old young male patient with progressive course over a period of 4 years with symptoms suggestive of multi-organ involvement.
\end{abstract}

Keywords: Alfa interferon, Erdheim-Chester syndrome, Interleukin-1, Proto-oncogene.

How to cite this article: Patil M, Jaiswal D. Erdheim-Chester Disease. J Med Sci 2015;1(3):55-57.

\section{Source of support: Nil}

\section{Conflict of interest: None}

\section{INTRODUCTION}

Erdheim-Chester is a disease characterized by the abnormal multiplication of a specific type of white blood cells called histiocytes or tissue macrophages (technically, this disease is termed a non-Langerhanscell histiocytosis). ${ }^{1}$ The disease affects patients of middle age. This disease causes an infiltration of lipidladen macrophages, multinucleated giant cell infiltrate of lymphocytes, and histiocytes in the bone marrow, which results in generalized sclerosis of the long bones. This disease was first described in 1930 by the Austrian pathologist, Jakob Erdheim, and the American pathologist, William Chester. ${ }^{2}$ This disease is at times difficult to diagnose. For these reasons, some feel the

\footnotetext{
${ }^{1}$ Professor, ${ }^{2}$ Resident

1,2Department of General Medicine, RajaRajeswari Medical College and Hospital, Bengaluru, Karnataka, India

Corresponding Author: Mamatha Patil, Professor, Department of General Medicine, RajaRajeswari Medical College and Hospital Bengaluru, Karnataka, India, e-mail: dr.mamatharamesh@ yahoo.com
}

disease could be under-diagnosed and may not be as rare as thought of.

\section{CASE REPORT}

A 26-year-old male who presented with complaints of difficulty in breathing noticed increasing dyspnea on exertion and orthopnea, which had progressively worsened over the past 3 years. He also experienced drooping of eyelid on the right side with decreased vision since 1 month. The drooping of eyelid was insidious in onset, progressive in nature without diurnal variation. He had a similar illness of drooping of right eyelid with double vision 2 years back. A history of hearing loss, more in the right than the left, ear pain, ear discharge, headache, bone and joint pain was present, along with giddiness and intermittent tinnitus, difficulty in speech, on and off since 3 years. The patient was on steroid treatment, from which he got relief for 2 to 3 months. There was no history of fever, vomiting, and seizure. On examination, patient was oriented and conscious, alert, afebrile. Extraocular movement of the right eye was restricted in all the directions except abduction. Left eye was normal in movement in all the directions. Bilateral papilloedema of the fundus was present. Right pupil was $4 \mathrm{~mm}$ and fixed, left was $2 \mathrm{~mm}$ and reactive. Vision acuity for right was 6/12 and for left 6/12. Bilateral conductive deafness was present. Motor and sensor systems were normal. Cardiovascular system(CVS), respiratory, perabdomen on clinical examination were normal. All blood investigations were in normal range. Lumbar puncture was done and csf analysis showed glucose- 68 , protein- 48 , chloride-126, and cell count-14 cell (all are lymphocytes). Pulmonary function testing showed restrictive lung disease. X-ray posteroanterior (PA) view of the chest X-ray (Figs 1 and 2) showed diffuse bilateral infiltrate of the lungs. Cerebrospinal fluid (CSF) antimyobacterial and anticysternal antibodies were negative by ELISA method. Cerebrospinal fluid VDRL-was non-reactive. Serum RA was negative. Serum ANA was also negative. Bone marrow histology showed complete replacement of the fatty marrow by a variable amount of fibrous tissue, prominent proliferation of foamy histiocytes, and multinucleated forms. Audiometry revealed profound hearing loss on the right side, bilateral middle ear pathology, and left moderate conductive hearing loss. Magnetic resonance imaging (MRI) scan brain in 2010 showed (Fig. 3) T2 hypointense thick nodular lesion seen along the dorsal aspect of cliws 


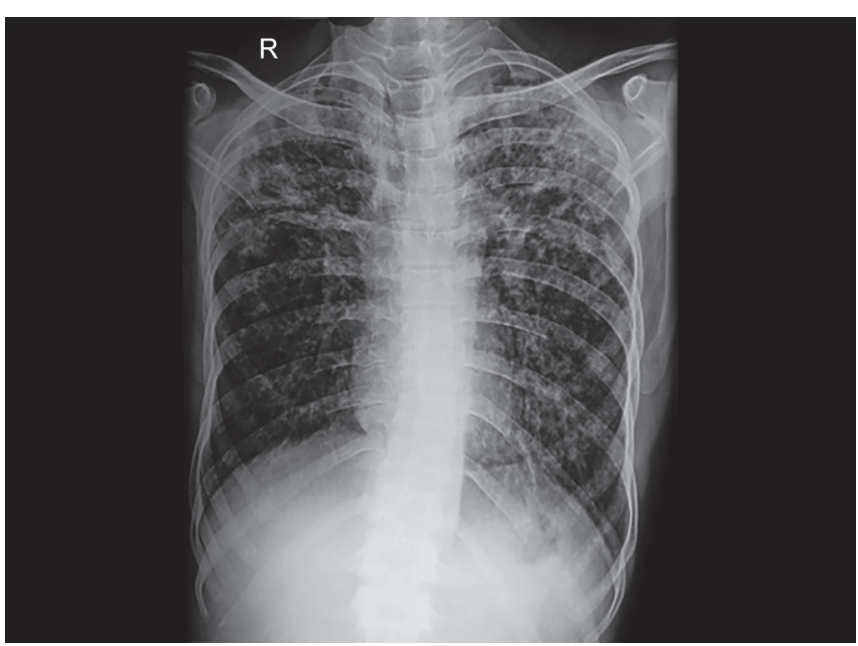

Fig. 1: X-ray posteroanterior view of the chest showing multiple bilateral infiltrates

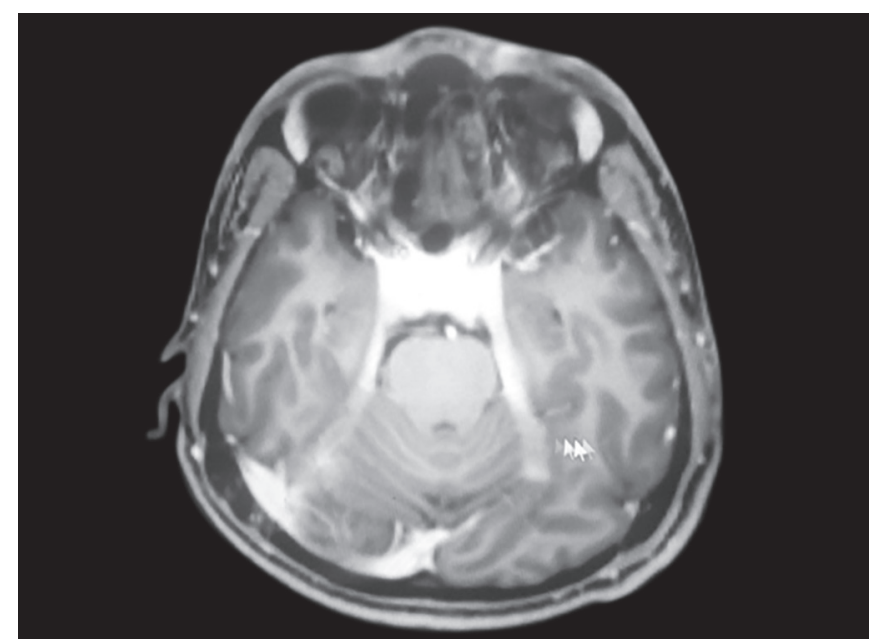

Fig. 3: Magnetic resonance imaging brain scan of 2010, suggestive of pachymeningitis involving sellar parasellar orbital apex and clivus

reaching down to sinus and orbital apex; lesion is isotense on TIWI and shows intense homogenous enhancement following in contrast. In 2014, there is evidence of thickened, nodular, T2 hypointense pachymeninges, showing intense contrast enhancement along the clivus, tentorium, sella, and middle cranial fossa base extending up to optic canals and orbitals fissures. These lesions are showing evidence of blooming on susceptibility weighted imaging.

\section{DISCUSSION}

Erdheim-Chester disease (ECD), a non-Langerhans form of histiocytosis, is a multisystemic disease characterized by various manifestations, such as skeletal involvement with bone pain, exophthalmoses, diabetes insipidus, renal impairment, and central nervous system (CNS) and/or cardiovascular involvement. ${ }^{1,3,4}$ Its prevalence is not known. Since 1930, more than 500 cases have been reported in the literature. Incidence is $<15$ in pediatric patients.

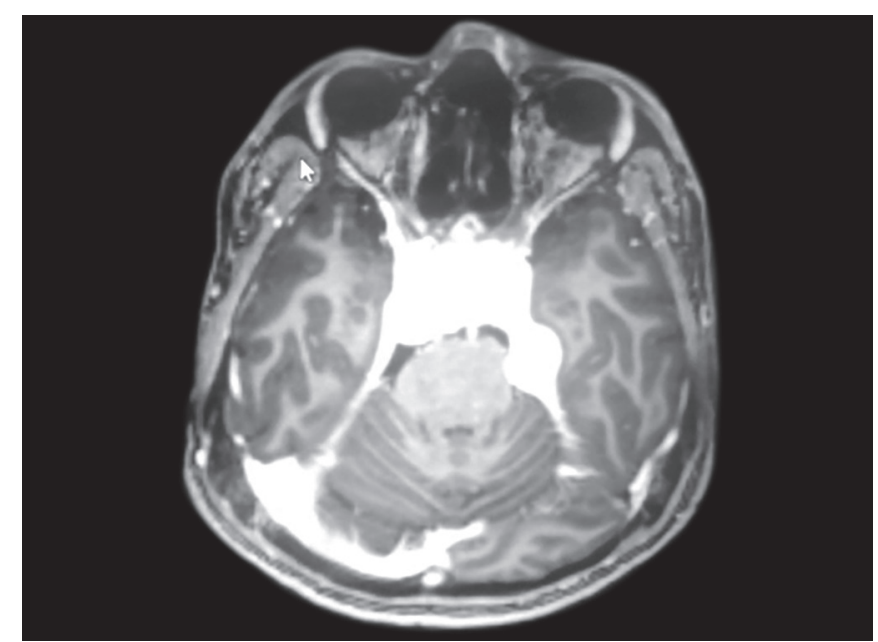

Fig. 2: Magnetic resonance imaging done in year 2014. Imaging shows nodular thickening and enhancement of the skull base pachymeninges

Erdheim-Chester disease usually presents in adults aged 40 to 60 with a 3:1 male to female ratio. ${ }^{3}$ Clinical course varies from asymptomatic to multisystemic, life-threatening forms. The pathognomonic feature of ECD is osteosclerosis of the long bones manifesting as bone pain, mainly affecting the distal lower limbs ( $50 \%$ of cases). Infiltration of the pituitary gland leads to diabetes insipidus and rarely hyperprolactinemia and gonadotropin insufficiency. ${ }^{5}$ Symptoms include fever, weakness, and weight loss. ${ }^{6}$ If other organs are filtrated, it leads to intracranial hypertension, exophthalmos, papilledema, adrenal insufficiency, xanthelasmas, and skin lesions. Central nervous system involvement can cause cerebellar and pyramidal syndromes, headaches, seizures, cognitive impairment, cranial nerve palsies, and sensory disturbances. ${ }^{74}$ "Coated aorta" is frequent cardiovascular involvement. Involvement of renal arteries can lead to renovascular hypertension. Involvement of pericardium may be complicated by a cardiac tamponade. In the cardiovascular system, pseudotumoral infiltration of the right atrium can occur. Dyspnea, due to infiltration of the lung, can lead to dyspnea. Pseudo retroperitoneal fibrosis can cause bilateral hydronephrosis.

This disease is of unknown etiology and is thought to be either due to reactive or neoplastic pathology. Elevated levels of interferon-alpha (IFN-alpha), interleukin (IL)-7, IL-12, and monocyte chemo attractant protein-1 and decreased levels of IL-4 found in ECD patients support an associated systemic immune Th-1 oriented perturbation. ${ }^{8,9}$ It is shown recently that mutations in the BRAF protooncogene occur in $>$ more than $50 \%$ of ECD cases. This adds another hypothesis to the pathophysiology of ECD. ${ }^{10}$ The hallmark histological finding is the xanthogranulomatous or xanthomatous infiltration of tissues with histiocytes. We may find CD68-positive and CD1a-negative ${ }^{6,11}$ on immunohistochemical staining of a biopsy sample. Bone 
X-rays usually display bilateral and symmetric cortical osteosclerosis of the long bones, while technetium $99 \mathrm{~m}$ bone scintigraphy shows almost constantly evidence of symmetric and abnormally strong labeling of the distal ends of the long bones of the lower limbs (and sometimes the upper limbs). ${ }^{12}$ Abdominal computed tomography (CT) scan may show a "hairy kidney" appearance (in $50 \%$ ), which can be biopsied. Langerhans' cell histiocytosis, Rosai-Dorfman disease, Takayasu arteritis, Wegener's granulomatosis, primary hypophysitis, chronic recurrent multifocal osteomyelitis malignancies, neurosarcoidosis, mycobacterial infections, and metabolic disorders are included in the differential diagnosis of this rare condition.

Administration of standard or pegylated IFN-alpha for all forms of ECD is the first line of treatment, with higher doses ( 9 million units, 3 times per week) on a long-term basis are required for those with CNS and cardiac localizations. ${ }^{13}$ To alleviate the bone pain, bisphosphonates are given. Drug of choice for those with orbital involvement is Cladribine and is useful in resistant. In mild forms of ECD in patients where IFNalpha was ineffective, Anakinra can improve symptoms. Infliximab and vemurafenib have been used recently, with some success. Vemurafenib is very promising for patients with a BRAFV600 mutation. To assess the disease activity, PET scans are usually recommended. This disease has a variable prognosis and is very poor in those with CNS involvement. The mean survival after diagnosis was 19.2 months, before the treatment with IFN-alpha. Now with IFN-alpha treatments, the mortality rate has come down to $26 \%$, and 5 -year survival has gone up to $68 \%$.

\section{CONCLUSION}

Erdheim-Chester disease, although a rare and orphan disease, has been overlooked and numerous new cases are currently being diagnosed because of better general knowledge of this histiocytosis. First-line treatment is IFN-alpha. Recently, a unique cytokine signature that may provide further clues to understand the pathogenesis of ECD as well as provide new tools for diagnosis and targeted therapy has been described.

\section{REFERENCES}

1. Arnaud L, Hervier B, Neel A, Hamidou MA, Kahn JE, Wechsler B, Perez-Pastor G, Blomberg B, Fuzibet JG, Dubourguet $\mathrm{F}$, et al. CNS involvement and treatment with interferon-alpha are independent prognostic factors in Erdheim-Chester disease: a multicenter survival analysis of 53 patients. Blood 2011 Mar;117(10):2778-2782.

2. Mazor RD, Manevich-Mazor M, Shoenfeld Y. ErdheimChester Disease: a comprehensive review of the literature. Orphanet J Rare Dis 2013 Sep;8(1):1375.

3. Volpicelli ER, Doyle L, Annes JP, Murray MF, Jacobsen E, Murphy GF, Saavedra AP. Erdheim-Chester disease presenting with cutaneous involvement: a case report and literature review. J Cutan Pathol 2011 Mar;38(3):280-285.

4. Drier A, Haroche J, Savatovsky J, Godeneche G, Dormont D, Chiras J, Amoura Z, Bonneville F. Cerebral, facial, and orbital involvement in Erdheim-Chester disease: CT and MR imaging findings. Radiology 2010 May;255(2):586-594.

5. Carpinteri R, Patelli I, Casanueva FF, Giustina A. Pituitary tumors: inflammatory and granulomatous expansive lesions of the pituitary. Best Pract Res Clin Endocrinol Metab 2009 Oct;23(5):639-650.

6. Veyssier-Belot C, Cacoub P, Caparros-Lefebvre D, Wechsler J, Brun B, Remy M, Wallaert B, Petit H, Grimaldi A, Wechsler B, et al. Erdheim-Chester disease. Clinical and radiologic characteristics of 59 cases. Medicine 1996 May;75(3):157-169.

7. Clerico A, Ragni G, Cappelli C, Schiavetti A, Gonfiantini M, Uccini S. Erdheim-Chester disease in a child. Med Pediatr Oncol 2003 Dec;41(6):575-577.

8. Tran TA, Pariente D, Lecron JC, Delwail A, Taoufik Y, Meinzer U. Treatment of pediatric Erdheim-Chester disease with interleukin-1-targeting drugs. Arthritis Rheum 2011 Dec;63(12):4031-4032.

9. Haroche J, Amoura Z, Trad SG, Wechsler B, Cluzel P, Grenier PA, Piette JC. Variability in the efficacy of interferon-alpha in Erdheim-Chester disease by patient and site of involvement: results in eight patients. Arthritis Rheum 2006 Oct;54(10):3330-3336.

10. Tran TA, Fabre M, Pariente D, Craiu I, Haroche J, Charlotte F, Eid P, Durrbach A, Taoufik Y, Kone-Paut I. Erdheim-Chester disease in childhood: a challenging diagnosis and treatment. J Pediatr Hematol Oncol 2009 Oct;31(10):782-786.

11. Haroche J, Arnaud L, Amoura Z. Erdheim-Chester disease. Curr Opin Rheumatol 2012 Jan;24(1):53-59.

12. Song SY, Lee SW, Ryu KH, Sung SH. Erdheim-Chester disease with multisystem involvement in a 4-year-old. Pediatr Radiol 2011 May;42(5):632-635.

13. Javier M, Filip J, Philip R, Cohen, Razelle K. Erdheim-Chester Disease: Characteristics and Management; Mayo Clin Proc 2014;89(7):985-996 